\title{
Neonatal arterial ischemic stroke and limb ischemia - clinical course and risk factors analysis
}

\author{
Ilona Kopyta ${ }^{1}$, Iwona Maruniak-Chudek ${ }^{2}$, Anna Balcerzyk ${ }^{3}$, Iwona Żak ${ }^{3}$, \\ Zbigniew Olczak ${ }^{4}$, Patrycja Sodowska ${ }^{5}$ \\ ${ }^{1}$ Department of Paediatrics and Developmental Age Neurology, Chair of Paediatrics, School of Medicine in Katowice, \\ Medical University of Silesia, Katowice, Poland \\ ${ }^{2}$ Department of Intensive Care and Neonatal Pathology, Chair of Paediatrics, School of Medicine in Katowice, \\ Medical University of Silesia, Katowice, Poland \\ ${ }^{3}$ Department of Biochemistry and Medical Genetics, School of Health Sciences in Katowice, \\ Medical University of Silesia, Katowice,Poland \\ ${ }^{4}$ Division of Diagnostic Imaging and Interventional Radiology, Upper Silesian Child's Health Center, Katowice, Poland \\ ${ }^{5}$ Department of Gyneacology and Obstetrics, Chair of Women's Health, School of Health Sciences in Katowice, \\ Medical University of Silesia, Katowice, Poland
}

\section{INTRODUCTION}

According to an up-to-date classification, the perinatal stroke refers to neurological symptoms presented by an infant around the time of birth due to vascular pathology, and confirmed by neuroimaging.

It's incidence is estimated at 1/1,600 to 5,000 live births.

Most of the children present early symptoms such as recurrent focal seizures, features of encephalopathy with generalized hypotonia, lethargy and feeding difficulties. In about $40 \%$ of the children the diagnosis is delayed.

The perinatal stroke may occur as a result of a co-incidence of maternal, placental, and fetal risk factors.

The authors present an extremely rare case of a girl born with both ischemic stroke and right upper limb ischemia. To the authors' knowledge this is the eleventh case described in the literature and the only one with the combination of the described risk factors.

\section{CASE REPORT}

The female newborn was born at 35 weeks gestation by emergency caesarean section to a young woman (second pregnancy and labor). Her birth weight was 2760 grams and her clinical condition estimated by Apgar score was moderate but quickly improved (Apgar score 7/8). Physical evaluation revealed right upper ischemia (Figure 1).

Imaging revealed no blood flow in the right humeral artery and in both carotid arteries (Figure 2 ) and acute is- chemic stroke of the right hemisphere was (Figure 3). The child was treated with heparine and subsequently with Acenokumarol. Necrotic skin area covered more than half of the forearm treated with antibiotics and surgery with good outcome (Figure 4). Radial nerve paralysis symptoms still persisted but weak active movements of the right hand's fingers were observed. There were no seizures detected. Chronic pain syndrome was treated with analgesics and laser-therapy with a satisfactory effect.

The traditional risk factors of ischemic stroke, serum lipids and lipoprotein (a) elevated levels, anticardiolipin antibodies

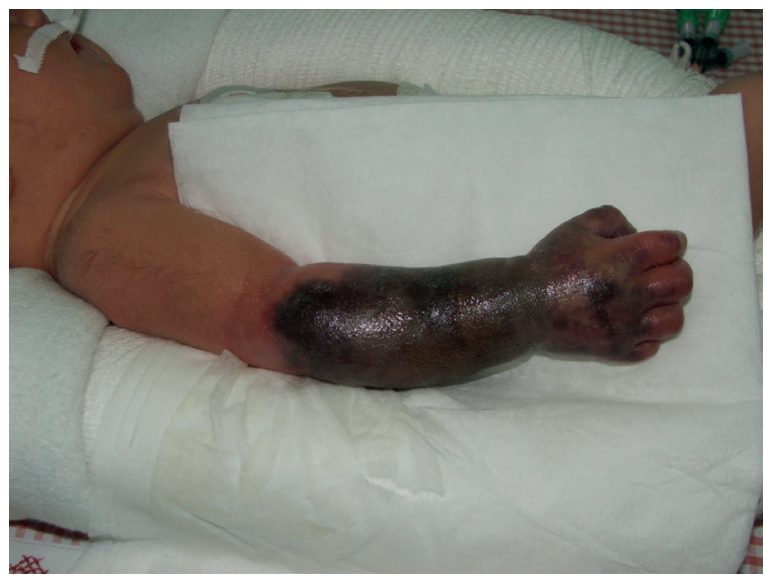

Figure 1. Right upper limb ischemia with skin necrosis 


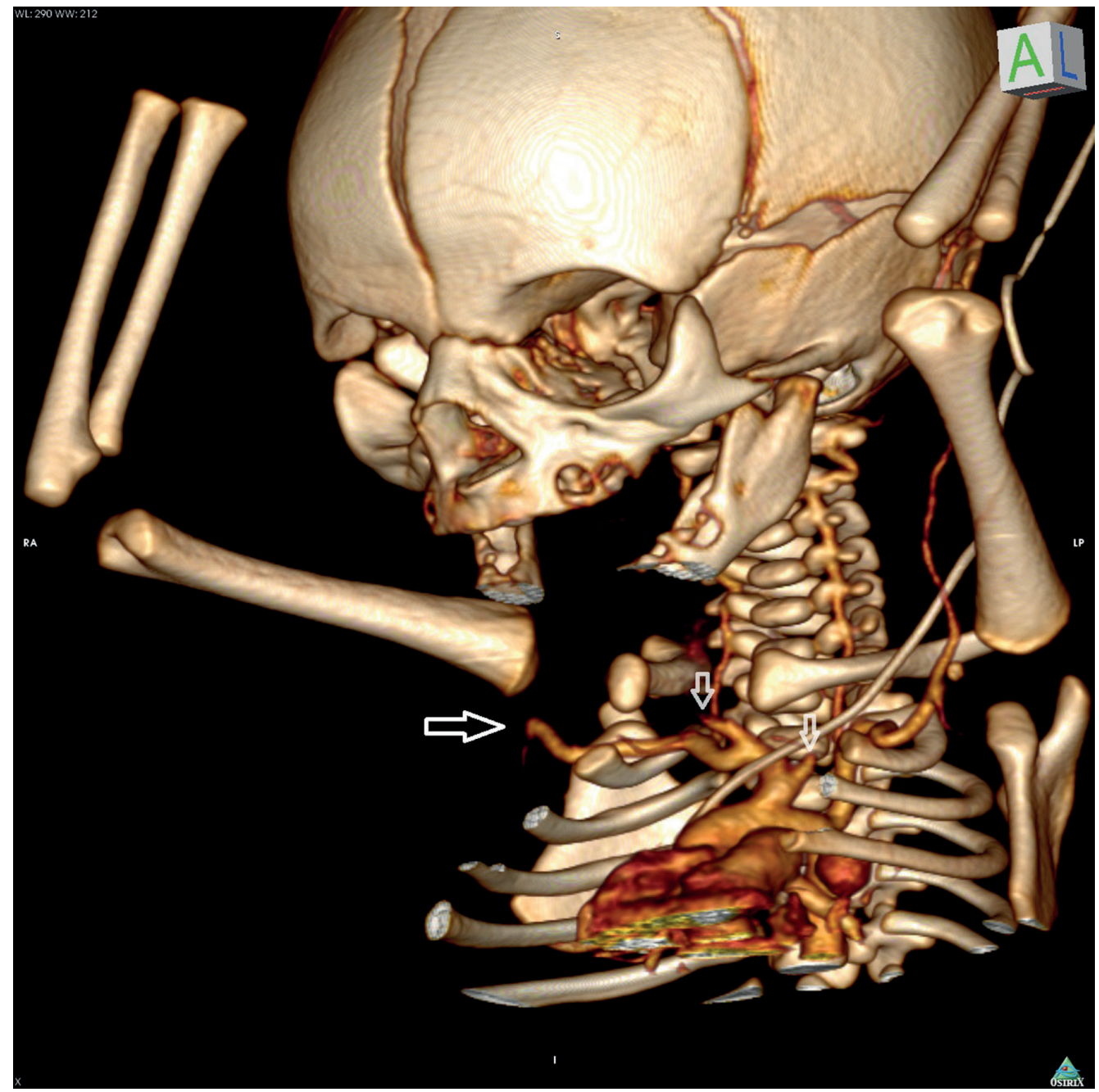

Figure 2. CT angiography, 3D VR reconstruction indicates occlusion of right subclavian artery and both CCA's. CT - computed tomography; VR — volume rendering; CCA — common carotid artery

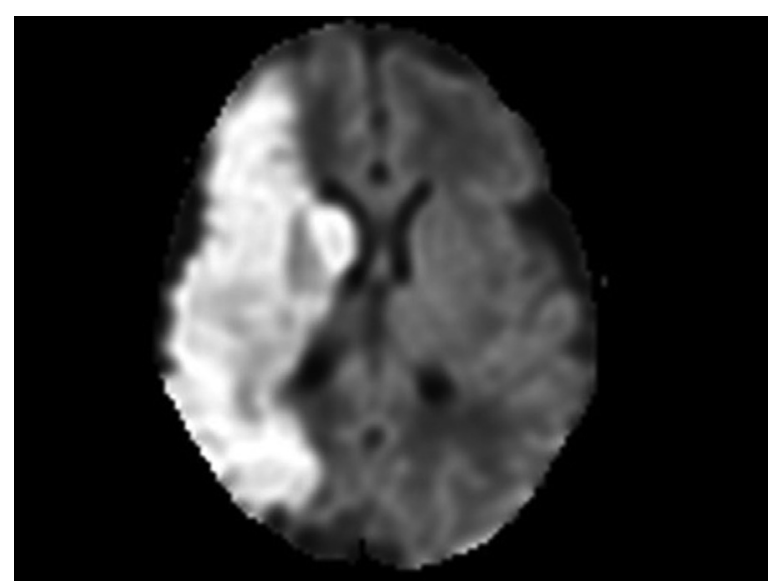

Figure 3. Head MRI, DWI sequence, ischemia in the right MCA territory; $\mathrm{MRI}$ - magnetic resonance imaging; DWI — diffusion weighted imaging; MCA — middle cerebral artery

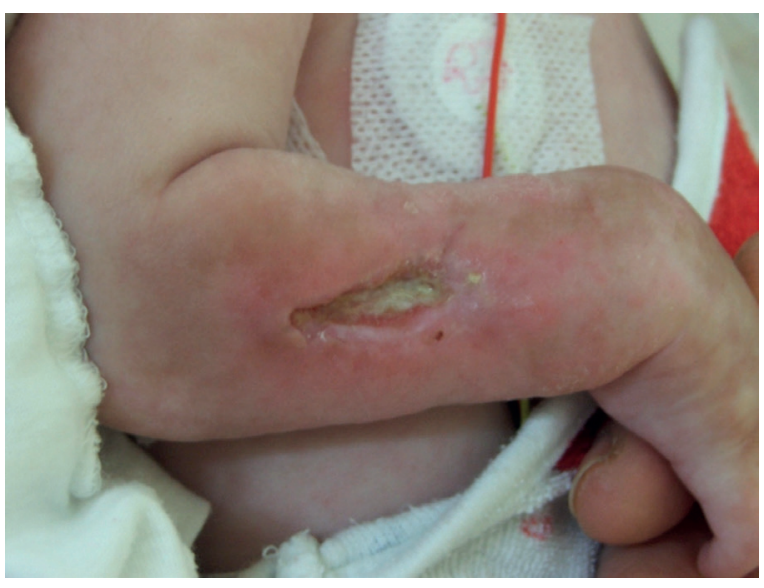

Figure 4. Right forearm after treatment 
in both IgG and IgM classes were excluded. Cytomegalovirus or herpes virus congenital infection was also excluded.

Protein $\mathrm{C}$ activity was decreased and the activity of protein $\mathrm{S}$ was on the borderline; the antithrombin III activity was normal.

The genetic analysis revealed the presence of the factor V Leiden mutation and TT homozygosity of the MTHFR gene (C667T polymorphism).

The girl is currently at the age of three and half years old. The social contact with the child is adequate to the age. On neurological examination she presents left-sided spastic hemiparesis and flaccid paresis of the right upper-extremity, with better motor function of the right-side of the body. The right upper limb is slimmer than the left one, large areas of scarifications are present, and the right hand is in palmar flexion.

\section{DISCUSSION}

In most of the neonatal stroke patients with mild clinical presentation the diagnosis is delayed. Both carotid arteries were occluded so blood supply was carried by verterbral arteries.

We conclude that neuroimaging should be considered for diagnosing a potential co-existence of brain and peripheral ischemia even if symptoms are not evident.

In our case, we managed to save the limb and avoid amputation, which was the case in four out of ten of the children described. 